\title{
ANÁLISIS DE DIVERSIDAD DEL COMPLEJO "GALLINA CIEGA" (COLEOPTERA: MELOLONTHIDAE) EN DOS SISTEMAS DE PRODUCCIÓN TRADICIONAL DE MAÍZ EN LA REGIÓN PURHÉPECHA, MICHOACÁN
}

\author{
Silvia Esperanza PÉrez-Agis', Miguel Ángel Morón², \\ Miguel Bernardo NÁJERA-RINCóN ${ }^{3}$, Edmundo LóPEZ-BARBOSA ${ }^{1}$ \\ y Marcelino VÁzQUEZ-GARCí ${ }^{4}$ \\ ${ }^{1}$ Facultad de Biología, Universidad Michoacana de San Nicolás de Hidalgo, Edificio R, \\ Planta Baja, Ciudad Universitaria, Av. J. Mújica s/n Col. Felicitas del Río. C. P. 58030, \\ Morelia, Michoacán, MÉXICO.grupo_marialuisam@yahoo.com.mx; \\ edmundocarlos@hotmail.com \\ 2 Departamento de Biología de Suelos, Instituto de Ecología, A.C. Apdo. Postal 63, C. P. \\ 91000 Xalapa, Veracruz, MÉXICO. miguel.moron@inecol.edu.mx \\ ${ }^{3}$ Campo Experimental Uruapan, Instituto Nacional de Investigaciones Agropecuarias y \\ Forestales URPAC. Av. Latinoamericana No. 1101 Col. Revolución C. P. 60500, Uruapan, \\ Michoacán, MÉXICO.minaj47@hotmail.com \\ ${ }^{4}$ Centro de Investigaciones en Parasitología Agrícola CUCBA Universidad de Guadalajara, \\ Km 15.5 Carr. Guadalajara-Nogales C. P.45100. Predio Las Agujas, Nextipac, Zapopan, \\ Jalisco, MÉXICO. mvazquez@cucba.udg.mx
}

\section{RESUMEN}

\begin{abstract}
En algunas regiones del Estado de Michoacán se considera que las larvas de coleópteros Melolonthidae conocidas como "gallina ciega" son una de las principales plagas rizófagas del maíz. El presente artículo aporta elementos sobre la composición del Complejo Gallina Ciega (CGC) en la Región Purhépecha en dos condiciones de cultivo contrastante, y relaciona la abundancia de las especies y su distribución anual con el posible impacto en el cultivo de maíz. Para el estudio se realizó un muestreo mensual por 10 meses, en dos localidades: la comunidad indígena San Felipe de los Herreros de la Meseta Purhépecha y el ejido San Francisco Uricho del Lago de Pátzcuaro. Los resultados obtenidos son: a nivel comunitario el CGC de ambas localidades registró una riqueza de 6 especies, con sus variantes en cada parcela. Las especies del CGC en el ejido San Francisco Uricho fueron: Macrodactylus sp., Anomala sp 2, Isonychus arizonensis, Phyllophaga sp. 4 y P. dentex; y en San Felipe de los Herreros fueron: Macrodactylus sp., Diplotaxis sp., Anomala punctatipennis, Anomala sp 1, P. vetula y P. platyrhina. Por su distribución mensual, en Uricho, las especies que podrían representar mayor riesgo para el cultivo de maíz son Phyllophaga dentex, y Phyllophaga sp. 4. Mientras que en San Felipe es P. platyrhina. Por su diversidad el modelo de distribución de abundancia en Uricho corresponde a poblaciones empobrecidas en donde la especie mas dominante es Macrodactylus sp., con un índice de equitatividad bajo de 0.53 en promedio. Mientras que en San Felipe, corresponde a una curva de
\end{abstract}


poblaciones mas equilibradas, en este caso debido a Macrodactylus sp. y P. platyrhina, con un índice de equitatividad de hasta 0.84 en promedio.

Palabras clave: Diversidad, gallina ciega, Macrodactylus, Phyllophaga, Isonychus, Anomala.

\begin{abstract}
White grubs causes an important damage to maize crops in the Mexican state of Michoacan. The present study offer information on the composition of the white grub species complex (CGC) at Purhepecha Region in two different crop conditions and show some relations between the abundance of species and their annual activity in the maize fields. The study was made at San Felipe de los Herreros in the Purhepecha Plateau and San Francisco Uricho near Lake Pátzcuaro during 10 months. Analysis of diversity was made with Biological Biotools software. At community level, both places have 6 species richness. Species complex at Uricho were formed by Macrodactylus sp., Anomala sp 2, Isonychus arizonensis, Phyllophaga sp. 4 and P. dentex; the most harmful species for maize could be Phyllophaga dentex and Phyllophaga sp. 4. Species complex at San Felipe were formed by Macrodactylus sp., Diplotaxis sp., Anomala puncatipennis, Anomala sp 1, P. vetula and P. platyrhina; the most important species in maize fields was $P$. platyrhina. The abundance curve at Uricho shows poor populations, while at San Felipe the equitability is better.
\end{abstract}

Key words: Diversity, Macrodactylus, Phyllophaga, Isonychus, Anomala, white grubs

\title{
INTRODUCCIÓN
}

Numerosos autores (Espinosa-Islas, et al., 2005; Nájera, 2005; Aragón-García y Morón, 2000; Ramírez-Salinas et al., 2000;) han señalado que una de las principales plagas rizófagas edafícolas asociadas a diversos cultivos de importancia alimentaria e industrial son las larvas de coleópteros conocidas como "gallina ciega". También se ha señalado que estas larvas normalmente se presentan como un conjunto de diferentes especies, sin embargo, poco se ha descrito sobre las características de las poblaciones que componen el conjunto y su relación con los daños al cultivo. Actualmente se sabe que solo un pequeño grupo de especies, que han sabido aprovechar oportunamente las condiciones de monocultivo y debido a su agresividad se comportan como una de las principales plagas de maíz (Zea mays), papa (Solanum tuberosum), caña de azúcar (Saccharun officinarum), cacahuate (Arachis hypogea), chile (Capsicum anumm), fríjol (Phaseolus vulgaris) diversas hortalizas y flores de ornato (Morón (1997), entre las que destacan Phyllophaga ravida (Blanch), Cyclocephala lunulata (Burnm) y Anomala cincta (Bay) asociadas con gramíneas (Morón, 1996).

En el ámbito estatal se estima que en Michoacán se siembran 895,252 hectáreas con cultivos cíclicos, de esta superficie el maíz abarca 58\% (524,015 ha). La región centro occidente del Estado se reconoce como una de las principales zonas productoras de maíz (SEM, 2004), en esta zona se localiza la Región Purhépecha donde este cultivo es uno de los principales, el cual abarca $5 \%$ de la superficie 
dedicada a este grano. En la subregión Lago de Pátzcuaro y Meseta Purhépecha (11 municipios) abarca aproximadamente 39.9 mil ha, aproximadamente el 5\% del total, cifra que sólo corresponde a los productores que están inscritos en PROCAMPO 2002-03, por lo cual está por debajo del dato real (INEGI, 2004). El promedio regional de rendimiento en grano es de 1.9 ton/ha, lo que hace a la región autosuficiente y productora excedentaria. Para el ciclo 2001-02 se estimó aproximadamente un rendimiento de 1.9 ton/ha, que distribuido entre la población daría $282 \mathrm{~kg} /$ per capita /anual de cuatro municipios de la región (INEGI, 2004), lo que da un excedente en la producción de $157.2 \mathrm{~kg} /$ per capita/anual (Pérez- Agis, obs.pers.).

El cultivo se desarrolla en condiciones de temporal, en suelos profundos de origen volcánico donde aproximadamente $30 \%$ de la superficie es agricultura de montaña (Gutiérrez et al., 2003). Para el INIFAP una de las principales plagas rizófagas en México es la "gallina ciega", y las especies que se han reportado en la región templada de Michoacán asociadas al maíz incluyen 17 especies de Phyllophaga, tres especies de Diplotaxis, cuatro especies de Macrodactylus, dos especies de Anomala, tres especies de Cyclocephala e Isonychus arizonensis (Howden), entre las que destacan por su importancia Phyllophaga ravida (Blanch.), P. vetula (Horn), $P$. dentex (Bates), P. misteca (Bates), P. rubella (Bates), C. lunulata y Anomala inconstans (Burmeister), entre otras (Nájera, 1998).

El objetivo del presente artículo consiste en aportar información sobre la composición del Complejo Gallina Ciega (CGC) en la Región Purhépecha en dos condiciones de cultivo contrastante, y relacionar la abundancia de las especies y su distribución anual al posible impacto en el cultivo de maíz.

\section{MATERIALES Y MÉTODOS}

\section{Ubicación del área de estudio y sistema de producción}

El estudio se realizó en dos localidades, una correspondiente a la subregión conocida como Meseta Purhépecha y la otra en la subregión Lago de Pátzcuaro, la primera es la comunidad indígena San Felipe de los Herreros, Municipio de Charapan, localizada a $102^{\circ} 10^{\prime} 54^{\prime \prime}$ longitud Oeste y $19^{\circ} 37^{\prime} 00^{\prime \prime}$ latitud Norte a una altitud de $2220 \mathrm{msnm}$ y la segunda es el ejido San Francisco Uricho, Municipio de Erongaricuaro localizado a $101^{\circ} 42^{\prime} 58^{\prime \prime}$ longitud Oeste y $19^{\circ} 34^{\prime} 20^{\prime \prime}$ latitud Norte, a una altitud de $2040 \mathrm{msnm}$, respectivamente. Ambas localidades se seleccionaron debido a que i) el maíz es su principal cultivo, ii) ambas localidades presentan condiciones agroecológicas similares, iii) el tipo de agricultura se clasifica como tradicional de subsistencia, aunque su sistema de producción es diferente. 
En cada localidad se seleccionaron cuatro parcelas agrícolas, con una superficie de 0.5 a 1 ha, sembradas de maíz criollo blanco cremoso durante el ciclo 2003-2004 de forma tradicional. En ambas localidades se verificó la presencia de "gallina ciega" en el periodo de barbecho (octubre 2003), también se confirmó mediante entrevistas informales a productores quienes ratificaron que la presencia de este insecto eventualmente provoca daños al cultivo. Para la caracterización del subsistema agrícola se aplicaron un total de 25 encuestas a productores agrícolas de ambas localidades.

\section{Muestreo entomológico}

Colecta preliminar. Para confirmar la presencia de "gallina ciega", en los meses de octubre y noviembre de 2003 se realizó una colecta de larvas en el periodo de barbecho mediante un transecto de $40 \mathrm{~m}$ atrás del tractor, en cinco parcelas del paraje agrícola en el que se trabajaría durante el ciclo 2004.

Muestreo mensual. Para registrar a las especies que se encuentran en el agroecosistema se programó un muestreo mensual, por diez meses a lo largo del año 2004. Cada mes, en cada parcela se tomaron cinco cepellones de 30 × 30 × $30 \mathrm{~cm}$ con los que se conformó una muestra de larvas.

Cría de larvas. Para la determinación de especies, de la colecta de 2003 se seleccionaron $50 \%$ de los individuos los cuales se confinaron en condiciones de cría, con vasos plásticos de $250 \mathrm{ml}$ con tierra de la parcela de procedencia y $5 \mathrm{ml}$ de agua semanal desde el mes de noviembre 2003 a mayo de 2004, de esta cría se obtuvieron adultos con los que se logró la identificación de cuatro especies, y cinco a nivel de morfoespecie. Posteriormente la identificación de larvas se asoció a los datos de la muestra de cría para determinar su especie. Se colectaron un total de 260 larvas, las cuales se fijaron en solución de Pampel y alcohol etílico al 70\%.

\section{Análisis de diversidad}

Para el cálculo de índices de diversidad se empleó el software estadístico Biological Tool Box versión 0.10 para Excel. Los índices que se seleccionaron fueron Equitatividad, Dominancia de Simpson, Diversidad de Simpson (1 - Dominancia), debido a que son mas sensibles a especies dominantes y su influencia en la diversidad. Equitatividad= H/Hmax; donde $\mathrm{H}$ es Diversidad de Shannon-Wiener; Hmax es Ln (S) del número de especies. Dominancia Simpson= $\mathrm{s}$ SUM (pi)^2; donde $\mathbf{s}$ es numero de especies; pi es proporción de los individuos por especie. Diversidad Simpson=1-C; donde C es el valor del índice de Dominancia de Simpson. 


\section{RESULTADOS}

\section{Características del subsistema de producción de maíz}

\section{Ejido San Francisco Uricho.}

La unidad de producción agrupa a los diferentes subsistemas de producción que la familia campesina articula para el aprovechamiento de los recursos naturales de los que dispone. En términos generales, la unidad está compuesta por un susbsistema agrícola, ganadería semi extensiva, pesca marginal y desarrollo de oficios como albañilería, artesanía textil y elaboración de tortilla para su venta en mercados regionales. Los oficios tienen la virtud de aportar ingresos económicos en efectivo que permiten a la familia hacer frente a gastos domésticos. Mientras que las actividades del sector primario aportan productos en especie para el autoconsumo y los excedentes sirven para su venta local y regional.

El subsistema de producción agrícola se caracteriza por hacer un uso intensivo de los escasos recursos que dispone cada agricultor (parcela promedio 3.5 ha) por lo que han desarrollado un sistema de asociación en el que el maíz se rota con cultivos forrajeros de invierno, los agricultores turnan el cultivo de dos gramíneas (Triticum turgidum, Avena sativa) y tres leguminosas (Vicia sativa, Vicia faba, Lens esculentum). Por otra parte han reducido los pasos de labranza y generado un esquema de labranza mínima a labranza cero que les ha permitido hacer un uso más eficiente de la escasa mano de obra, tierra y tiempo de cultivo. De tal manera que pueden lograr prácticamente dos cosechas al año. El rendimiento de maíz es de los mas altos de la región, 3 ton/ha, mientras que el de los de forrajes es medio, se calculó un porcentaje promedio de cobertura para la avena de $44 \%$, trigo $36 \%$ y ebo $63 \%$ (Ver Cuadro 1)

A pesar de que estos sistemas son dependientes del consumo de insumos químicos para el manejo fitosanitario y fertilización así como externos como tracción mecánica. Se les sigue considerando como modelo campesino debido a que se basa en la dependencia de mano de obra familiar, están orientados hacia el autoabasto familiar, emplean recursos base nativos (variedades criollas), los excedentes se dirigen hacia el mercado local y regional mas que nacional.

\section{Comunidad Indígena San Felipe de los Herreros.}

En este caso la unidad de producción agrupa al subsistema forestal, el subsistema agropecuario y el oficio de artesanía textil de las mujeres. Cabe señalar que el aprovechamiento forestal es el eje económico de la comunidad, los productos están orientados hacia el mercado nacional, esta actividad es la que mayor efectivo aporta a la comunidad (Gutiérrez, et al., 2003). 
Pérez Agis et al.: Diversidad del complejo "gallina ciega" (Coleoptera: Melolonthidae)

\section{Cuadro 1}

Determinantes agroecológicas de los sistemas producción de maíz en la Región Purhépecha, Michoacán.

\begin{tabular}{|c|c|c|c|}
\hline \multicolumn{2}{|c|}{ Agroecosistema } & $\begin{array}{l}\text { Sistema de año y vez } \\
\text { C.I. San Felipe de los Herreros } \\
\text { Subregión: Meseta Purhépecha }\end{array}$ & $\begin{array}{ll}\text { Sistema Año con Año } & \text { Ejido San } \\
\text { Francisco Uricho } & \\
\text { Subregión: Lago de Pátzcuaro } & \end{array}$ \\
\hline \multicolumn{2}{|c|}{ BIOFÍSICAS } & \multicolumn{2}{|c|}{$\begin{array}{l}\text { Clima subhúmedo con lluvias en verano. Temperatura media anual de } 16.6^{\circ} \mathrm{C} \text { a } 17.4^{\circ} \mathrm{C} \text {, altitud } 2200 \\
\text { a } 2400 \mathrm{msnm} \text {. Precipitación anual de } 1000 \text { a } 1029 \mathrm{~mm} \text {. Heladas tempranas de octubre a enero que } \\
\text { afectan el llenado de grano del cultivo. El granizo se presenta de } 2 \text { a } 4 \text { días al año acompañado de } \\
\text { viento durante el mes de junio y julio. Suel os Andosol, Andosol húmico y Cambisol, profundos y } \\
\text { pendiente mayor al } 5 \% \text {. }\end{array}$} \\
\hline \multicolumn{2}{|c|}{ TECNOLÓGICAS } & & \\
\hline \multirow{2}{*}{$\begin{array}{l}\mathrm{M} \\
\mathrm{A} \\
\mathrm{N} \\
\mathrm{E}\end{array}$} & $\begin{array}{l}\text { Cultivos: } \\
\text { especies y } \\
\text { variedades }\end{array}$ & $\begin{array}{l}\text { Maíz blanco criollo } \\
\text { Frijol criollo (Pheseolus vulgaris) }\end{array}$ & $\begin{array}{l}\text { Maíz criollo blanco } \\
\text { Cultivos de invierno semillas criollas: Vicia } \\
\text { sativa (ebol), Avena sativa (avena), Triticum } \\
\text { turgidum (trigo) }\end{array}$ \\
\hline & $\begin{array}{l}\text { Organización } \\
\text { cronológica } \\
\text { de los } \\
\text { cultivos }\end{array}$ & $\begin{array}{l}\text { Monocultivo y p equeñas superficies con policultivo } \\
\text { simultáneo: maíz + frijol } \\
\text { Crecimiento de arvenses nativas durante el periodo } \\
\text { de descanso }\end{array}$ & $\begin{array}{l}\text { Asociación en rotació n: Maíz - ebol; maíz - } \\
\text { avena; maíz - lenteja; maíz -trigo. }\end{array}$ \\
\hline \multirow{2}{*}{$\begin{array}{l}\mathrm{T} \\
\mathrm{R} \\
\mathrm{A}\end{array}$} & $\begin{array}{l}\text { Preparación } \\
\text { del terreno }\end{array}$ & $\begin{array}{l}\text { Barbecho mecánico en octubre } \\
\text { Rastreo mecánico en febrero }\end{array}$ & $\begin{array}{l}\text { Labranza reducida a un paso de rastra } 0 \\
\text { barbecho, mecánico en mayo }\end{array}$ \\
\hline & $\begin{array}{l}\text { Labores } \\
\text { culturales }\end{array}$ & $\begin{array}{l}\text { Surcado con tracción animal, siembra manual en } \\
\text { abril } \\
\text { Aporcado y escarda con tracción animal en julio y } \\
\text { septiembre }\end{array}$ & Surcado mecánico, siembra manual en junio \\
\hline \multirow{2}{*}{$\begin{array}{l}\text { S } \\
\mathrm{U} \\
\mathrm{E} \\
\mathrm{L} \\
\mathrm{O}\end{array}$} & Conservación & $\begin{array}{l}\text { Periodo de descanso de } 10 \text { meses } \\
\text { Cobertura de un } 50 \% \text { de restos de rastrojo }\end{array}$ & $\begin{array}{l}\text { Periodo de descanso un mes } \\
\text { Cultivos forrajeros con cobertura de hasta un } \\
70 \% \text { en el periodo de invierno }\end{array}$ \\
\hline & Fertilización & $\begin{array}{l}\text { Mixta } \\
\text { Químico a la siembra } \\
\text { Orgánica: Libre pastoreo por } 3 \text { meses, aplicación de } \\
\text { estiércol a la siembra. }\end{array}$ & $\begin{array}{l}\text { Química } \\
\text { A la siembra } 18-46-0 \\
\text { Al mes } 46-0-0\end{array}$ \\
\hline \multirow{3}{*}{$\begin{array}{l}\text { S } \\
\text { A } \\
\text { N } \\
\text { I } \\
\text { T } \\
\text { A } \\
\text { R } \\
\text { I } \\
\text { O } \\
\text { S }\end{array}$} & $\begin{array}{l}\text { Control de } \\
\text { malezas }\end{array}$ & $\begin{array}{l}\text { Deshierbe Manual y por densidad de siembra. } \\
\text { Principales malezas, herbáceas de diferentes } \\
\text { familias }\end{array}$ & $\begin{array}{l}\text { Escarda química (sal dietalonamina del ac. } 4 \text { - } \\
\text { amino-3,5,6-tricloropicolinico }+ \text { atrazina }(2- \\
\text { cloro-4-etilamino-6-isopropilamino } 1,3,5- \\
\text { triazina) } \\
\text { Principales malezas, gramíneas anuales }\end{array}$ \\
\hline & $\begin{array}{l}\text { Control de } \\
\text { plagas }\end{array}$ & $\begin{array}{l}\text { No hay una medida específica } \\
\text { Principales plagas (en orden de importancia): larvas } \\
\text { de Lepidoptera: Noctui dae (trozador), l arvas de } \\
\text { Coleoptera: Elateridae (alambre) y larvas de } \\
\text { Coleoptera: Melolonthidae (gallina ciega) }\end{array}$ & $\begin{array}{l}\text { Químico (Paration metílico: 0,0-Dimetil 0,p- } \\
\text { Nitrofenil Tiofosfato) } \\
\text { Principales plagas: larvas de Coleoptera: } \\
\text { Melolonthidae (gallina ciega), larvas de } \\
\text { Lepidoptera: Noctuidae (trozador) y larvas de } \\
\text { Coleoptera: Elateridae (alambre) }\end{array}$ \\
\hline & $\begin{array}{l}\text { Control de } \\
\text { enfermedades }\end{array}$ & No hay & Eventualmente problemas de roya en trigo \\
\hline \multicolumn{2}{|c|}{$\begin{array}{l}\text { Objetivo de la } \\
\text { producción }\end{array}$} & Autoconsumo & $\begin{array}{l}\text { Autoconsumo de maíz. Venta local y regional } \\
\text { de forrajes de invierno }\end{array}$ \\
\hline \multicolumn{2}{|c|}{ Tipo de mano de obra } & Familiar y rentada con remuneración en especie & Familiar y rentada con pago en efectivo \\
\hline \multicolumn{2}{|c|}{$\begin{array}{l}\text { Organización para la } \\
\text { producción }\end{array}$} & Sociedad de Producción Rural Tareris & $\begin{array}{l}\text { Incipiente organización de productores de } \\
\text { maíz orgánico }\end{array}$ \\
\hline \multicolumn{2}{|c|}{$\begin{array}{l}\text { Puntos débiles o } \\
\text { críticos }\end{array}$} & $\begin{array}{l}\text { Baja productividad, baja agrodiversi dad, pobre } \\
\text { manejo de la fertilidad de suelos }\end{array}$ & $\begin{array}{l}\text { Creciente dependencia de agroqu ímicos, e } \\
\text { impacto de plagas en maíz y frutales de la } \\
\text { región }\end{array}$ \\
\hline
\end{tabular}


El subsistema agropecuario regionalmente es conocido como año y vez el cual consiste en la alternancia de grandes parajes que se destinan a la agricultura después de un periodo sin cultivo de diez meses. Durante este periodo, de descanso, estas áreas se destinan al libre pastoreo por tres meses, después se dejan empastar hasta que en junio se vuelven a pastorear y en octubre inicia la preparación del terreno con el barbecho, en febrero el rastreo y en abril la siembra de maíz. Este sistema de manejo ha permitido que la ganadería sea parte integral del manejo para la recuperación de la fertilidad del suelo, aprovechamiento del rastrojo de maíz y pastos nativos (Ver Cuadro 1).

El sistema de año y vez es poco dependiente del consumo de insumos químicos así como externos como tracción mecánica. Se le considera como modelo campesino por basarse en el uso de mano de obra familiar, los productos están orientados hacia el autoabasto familiar, emplean recursos base nativos (variedades criollas y estiércol), los excedentes se dirigen hacia el mercado local y regional.

\section{Diversidad de larvas}

\section{Composición del Complejo Gallina Ciega (CGC)}

Ejido San Francisco Uricho. Debido a que en este ejido se cultivan forrajes de invierno (de noviembre a abril), como Avena sativa L. (avena), Triticum turgidum L. (trigo) y Vicia sativa L. (ebo), el muestreo se inició en diciembre de 2003 y continuó hasta diciembre 2004, se suspendió 3 meses durante el periodo de rastreo y siembra de maíz ya que el suelo estaba muy seco y no se registraba ningún insecto. Se colectaron 165 larvas, de las cuales se identificaron 124, esto es el $75 \%$ del total. Se registró un promedio de 0.75 larvas por cepellón.

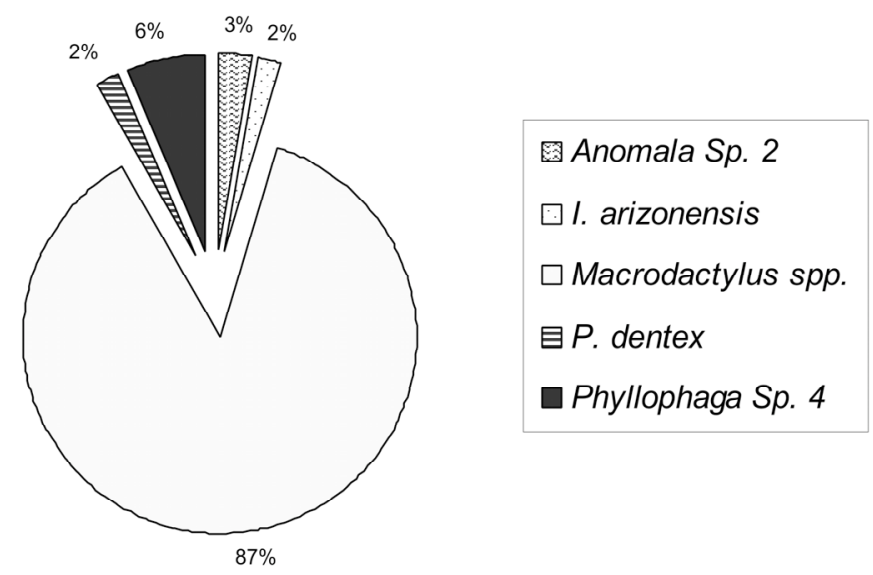

Figura 1

Composición CGC. Ciclo agrícola 2003-04. San Francisco Uricho, Mich. 
De la familia Melolonthidae, se determinaron larvas de 4 géneros con 5 especies, éstas fueron Macrodactylus sp. con 94 individuos identificados (87\%), Phyllophaga sp. 4 con 7 individuos (6\%), Anomala sp. 2 con 3 individuos (2\%), Phyllophaga dentex (Bates) con 2 individuos (2\%), e Isonychus arizonensis Howden con 2 individuos (2\%) (Fig. 1).

\section{Comunidad Indígena San Felipe de los Herreros.}

El muestreo inició en febrero de 2004 y continuó hasta diciembre del mismo año, debido a que durante el periodo de rastreo (diciembre a febrero) se presentan muy bajas temperaturas y casi no se registran individuos, así como durante los primeros meses después de la siembra (marzo). Se colectaron 95 larvas, de las cuales se identificaron 57 , esto es el $60 \%$ del total. Se registró un promedio de 0.30 larvas por cepellón.

De la familia Melolonthidae, se determinaron larvas de 4 géneros con 5 especies, éstas fueron Macrodactylus sp. con 32 individuos identificados (55\%), Phyllophaga platyrhina (Bates) con 12 individuos (21\%), Anomala sp. 1 con 6 individuos (11\%), Diplotaxis sp. con 5 individuos (9\%), y Anomala punctatipennis Blanch. con 2 individuos (4\%) (Fig. 2).

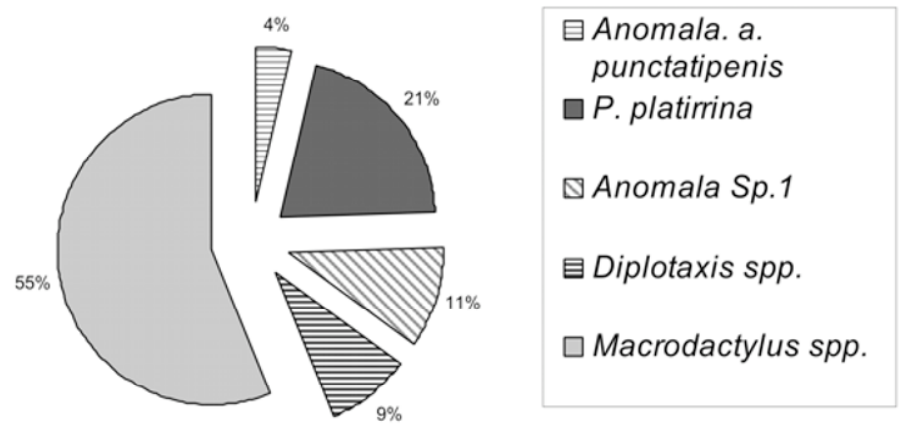

Figura 2

Composición CGC. Ciclo agrícola 2003-04 San Felipe, Mich.

\section{Distribución mensual y cobertura vegetal}

La distribución de las especies a lo largo del año y su relación con la cobertura vegetal, resulta especialmente importante para tratar de determinar cual de las especies puede ser la que probablemente ocasiona daños al cultivo de maíz o de invierno. 


\section{Ejido San Francisco Uricho.}

El género mas abundante es Macrodactylus sp. el cual se presenta a lo largo de todo el ciclo, sin registro en junio y julio, es especialmente abundante durante el periodo de invierno donde probablemente se alimenta y resguarda bajo los cultivos de invierno (Fig. 3). Bajo la cobertura de maíz, las especies coexisten, especialmente a partir de agosto a diciembre, en julio se registró Anomala sp 2, en agosto $I$. arizonensis y Phyllophaga sp. 4, ésta última se mantiene presente hasta octubre y finalmente de octubre a diciembre se registró $P$. dentex. Esta información sugiere que las especies que podrían afectar el desarrollo del cultivo de maíz son Macrodactylus sp. y Phyllophaga sp. 4. ya que se registraron a lo largo del periodo de crecimiento de maíz cuando su sistema radicular es mas tierno y turgente. Sin embargo, cabe señalar que los picos de abundancia de Macrodactylus son más altos durante el periodo de invierno.

Durante la colecta de octubre 2003, en el periodo de rastreo para la siembra de cultivos de invierno, en 5 parcelas se registraron 104 larvas de las cuales 38\% correspondieron a P. dentex, $29 \%$ a Macrodactylus sp., 25\% a I. arizonensis y $1.9 \%$ Phyllophaga sp.4. Esta información sugería que las dos especies de Phyllophaga podrían representar un riesgo para el maíz. Con el resultado del muestreo mensual se corrobora que Phyllophaga sp.4. es la que puede estar afectando al maíz (Fig. 3).
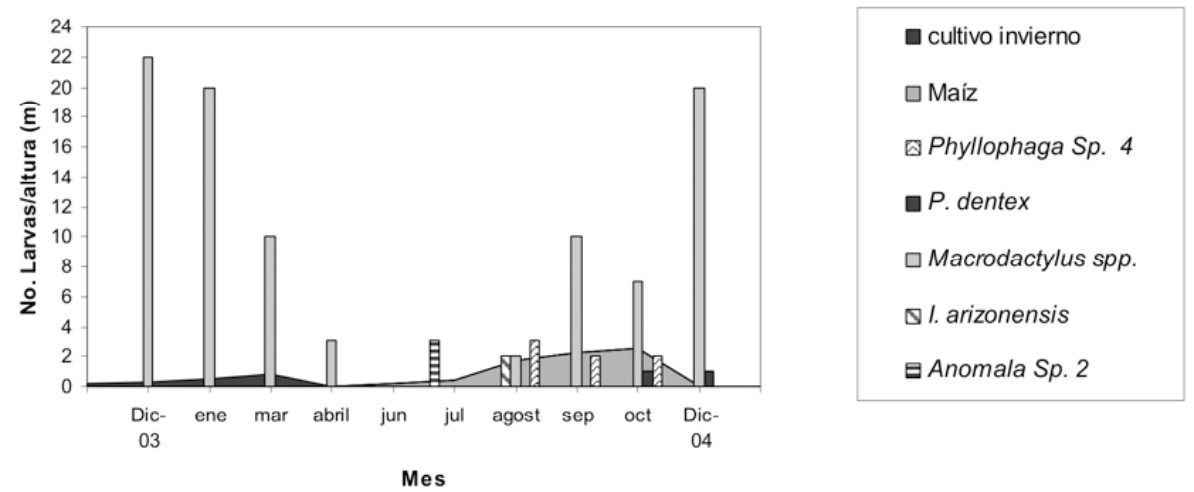

Figura 3

Distribución por especie y altura de cultivos. San Francisco Uricho, 2004.

\section{Comunidad Indígena San Felipe de los Herreros.}

El género mas abundante es Macrodactylus sp. el cual se presenta especialmente durante el periodo de invierno donde probablemente se resguarda bajo plantas arvenses que crecen durante el periodo de descanso (enero a octubre), (Fig. 4). En 
este mismo periodo coexisten tres especies mas, Anomala sp 1, Diplotaxis, Anomala puncatipennis y P. platyrhina. Bajo la cobertura de maíz, la especie más abundante y constante es $P$. platyrhina. Esta información sugiere que la especie que podría afectar el desarrollo del cultivo de maíz es Phyllophaga platyrhina.

Durante la colecta de octubre 2003, en el periodo de barbecho, se registraron 108 larvas en 5 parcelas, en este caso $61 \%$ correspondió a $P$. platyrhina, $22 \%$ a $P$. vetula y $14 \%$ a Anomala punctatipennis, esta información sugiere que al menos $P$. platyrhina puede ser la que provoca daños al cultivo de maíz lo que se corrobora con el muestreo mensual.

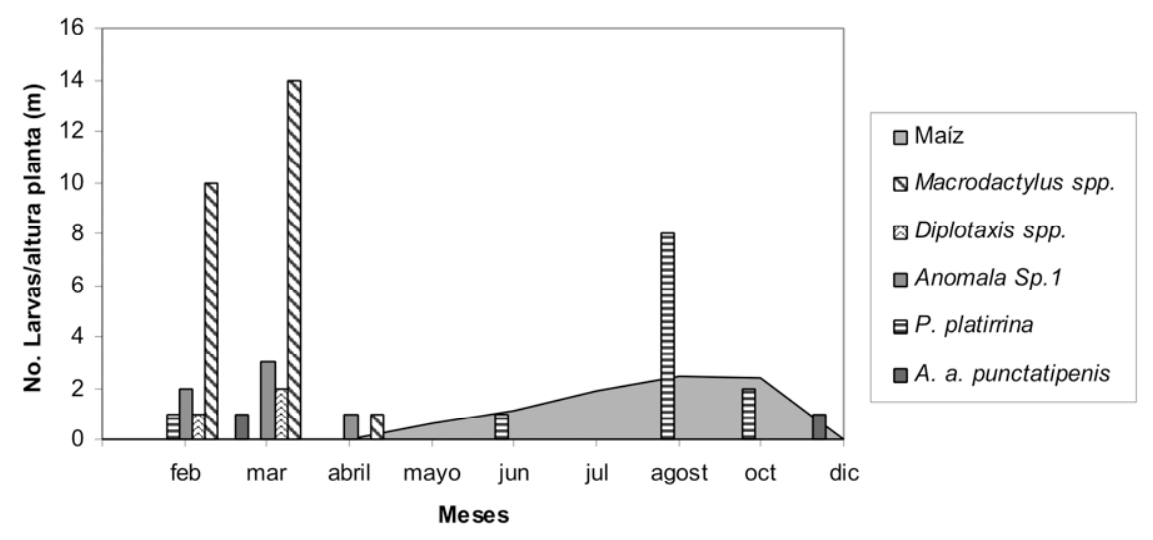

Figura 4

Distribución por especie y altura de maíz. San Felipe, 2004.

\section{Dominancia de especie por parcela}

En los resultados anteriores se muestra un panorama general de la distribución de las especies del CGC a nivel de localidad, sin embargo esta realidad no se aplica a cada parcela como a continuación se presenta. El análisis de diversidad consistió en el computo de lo individuos identificados por lo menos hasta género, su diversidad a nivel de parcela y representación proporcional a nivel de localidad.

\section{Ejido San Francisco Uricho.}

Como se muestra en los Cuadros 2 y 3 , la abundancia de individuos y la riqueza de especies son diferentes en cada parcela. La parcela con mayor riqueza de especies (5 especies) y mayor abundancia de larvas es u4. La parcela con menor índice de equitatividad y mayor índice de dominancia es u2, debido a la dominancia de Macrodactylus sp. por lo cual su índice de diversidad es también el menor. 


\section{Cuadro 2}

Abundancia por especie y parcela. San Francisco Uricho, 2004.

\begin{tabular}{|l|c|c|c|c}
\hline Especie/ Parcela & $\mathbf{u 1}$ & $\mathbf{u 2}$ & $\mathbf{u 3}$ & $\mathbf{u 4}$ \\
\hline I. arizonensis & 2 & & & 1 \\
\hline Macrodactylus & 10 & 31 & & 45 \\
\hline P. dentex & 1 & 1 & 3 & 17 \\
\hline Phyllophaga sp. 4 & & 4 & & 4 \\
\hline P. platyrhina & & 1 & & \\
\hline Anomala sp. 2 & & & 3 & \\
\hline
\end{tabular}

Cuadro 3

Diversidad por parcela. San Francisco Uricho, 2004.

\begin{tabular}{|l|r|r|r|r|r|}
\hline \multirow{2}{*}{ Parcela/Índice } & & & Diversidad de & \\
& & Dominancia de & Simpson (1 - & Total & Riqueza de \\
& Equitatividad & Simpson & Dominancia) & individuos & especies \\
\hline u1 & 0,6254 & 0,6213 & 0,3787 & 13 & 3 \\
\hline u2 & 0,4212 & 0,7151 & 0,2849 & & 37 \\
\hline u4 & 0,6133 & 0,4776 & 0,5224 & & 70 \\
\hline Uricho & 0,5336 & 0,5263 & 0,4737 & & 123 \\
\hline
\end{tabular}

La parcela con mayor índice de equitatividad es u1, sin embargo presenta un bajo índice de diversidad por la dominancia de Macrodactylus sp. La parcela u4 a pesar de que tiene un buen índice de equitatividad debido a la abundancia de 2 especies (Macrodactylus sp. y $P$. dentex) es la parcela que registra (en la opinión del productor) mayores daños por gallina ciega, que en este caso probablemente se debe al efecto de $P$. dentex en el cultivo de maíz. La parcela u3 fue la parcela con menos abundancia y diversidad ya que solo se registro $P$. dentex a partir del mes de septiembre. 
A nivel de localidad, la diversidad del CGC está dominado por Macrodactylus sp. en la Figura 5, donde se representa la abundancia relativa por especie, se describe una curva conocida como "serie geométrica", este patrón de distribución se presenta frecuentemente en ambientes pobres en especies o durante los primeras fases de sucesión o en condiciones ambientales deterioradas (Magurran, 1987).

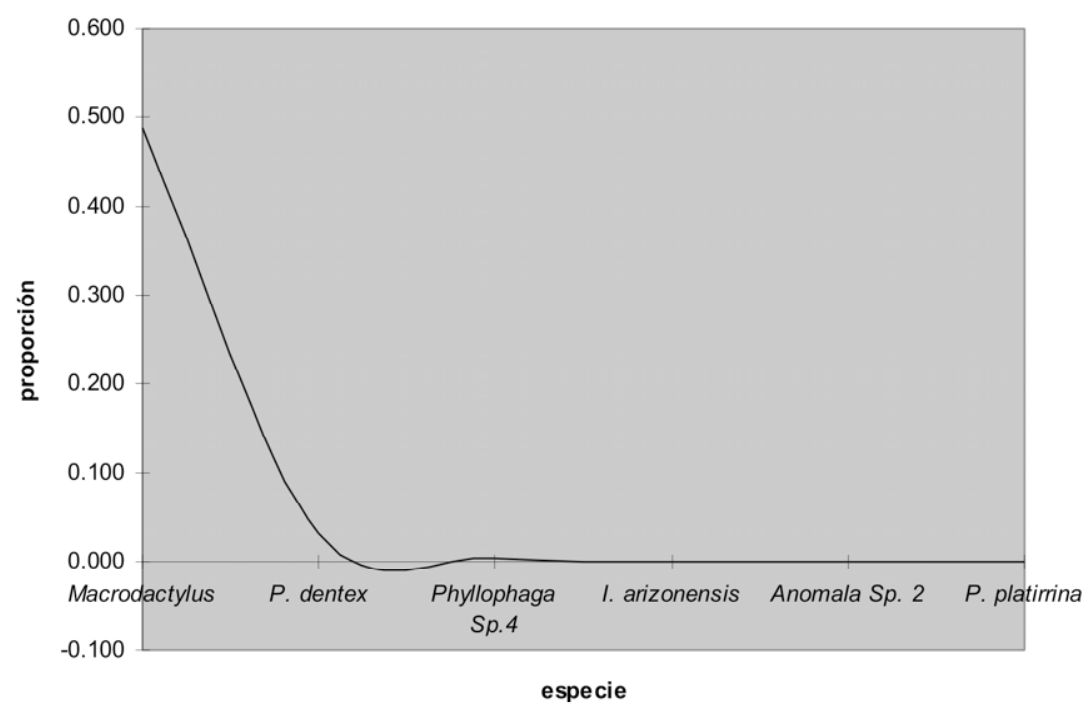

Figura 5

Abundancia relativa por especie. San Francisco Uricho, 2004

\section{Comunidad Indígena San Felipe de los Herreros}

Como se muestra en los Cuadros 4 y 5 la abundancia de individuos y la riqueza de especies se mantiene más constante. La parcela con mayor riqueza de especies (6 especies) es sf 2 , la cual a su vez presenta un bajo índice de dominancia y el más alto índice de diversidad, no es la más equitativa debido a la abundancia de $P$. platyrhina. La parcela mas equitativa es sf3, debido a que no hay dominancia de ninguna especie por lo que su índice de diversidad es el mas alto. La parcela menos equitativa es sf4, debido a la abundancia de P. platyrhina, por lo que su índice de dominancia es el mas alto y de diversidad es el mas bajo

A nivel de localidad, la diversidad del CGC está dominado por $P$. platyrhina $y$ Macrodactylus sp. en la Figura 6, se describe una curva conocida como "serie logarítmica", este patrón de distribución se presenta frecuentemente en ambientes en los que algunos factores controlan las condiciones ecológicas de la comunidad y son aprovechadas por pocas especies, las cuales dominan (Magurran, 1987). 


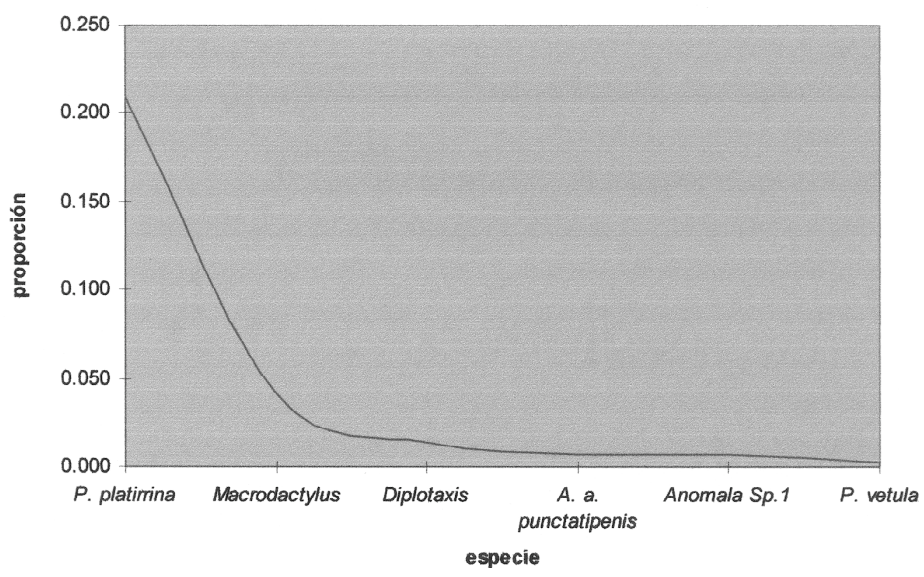

Figura 6

Abundancia relativa por especie. San Felipe, 2004.

\section{Cuadro 4}

Abundancia por especie y parcela. San Felipe de los Herreros, 2004.

\begin{tabular}{|l|c|c|c|c}
\hline Especie/ parcela & sf1 & sf2 & sf3 & sf4 \\
\hline P. platyrhina & 10 & 12 & 2 & 19 \\
\hline A. punclatipennis & 2 & 1 & 1 & 4 \\
\hline Macrodactylus & 8 & 5 & 4 & 2 \\
\hline P. vetula & 1 & 3 & & 1 \\
\hline Diplotaxis & 2 & 1 & 8 & \\
\hline Anomala sp.1 & & 5 & 2 & 1 \\
\hline
\end{tabular}


Pérez Agis et al.: Diversidad del complejo "gallina ciega" (Coleoptera: Melolonthidae)

\section{Cuadro 5}

Diversidad por parcela. San Felipe de los Herreros, 2004.

\begin{tabular}{|l|r|r|r|r|r|}
\hline Parcela/Índicc & Equitatividad & \multicolumn{1}{|l|}{$\begin{array}{l}\text { Dominancia de } \\
\text { Simpson }\end{array}$} & $\begin{array}{l}\text { Simpson (1 - } \\
\text { Dominancia) }\end{array}$ & $\begin{array}{l}\text { Total } \\
\text { individuos }\end{array}$ & $\begin{array}{l}\text { Riqueza de } \\
\text { especies }\end{array}$ \\
\hline sf1 & 0,8019 & 0,3270 & 0,6730 & 23 & 5 \\
\hline sf2 & 0,8223 & 0,2812 & 0,7188 & 27 & 6 \\
\hline sf3 & 0,8484 & 0,3080 & 0,6920 & 17 & 5 \\
\hline sf4 & 0,6009 & 0,5254 & 0,4746 & 27 & 5 \\
San Felipe & 0,8413 & 0,2811 & 0,7189 & 94 & 6 \\
\hline
\end{tabular}

\section{DISCUSIÓN}

Según Morón (1996) algunos factores que influyen en la dispersión y aumento de poblaciones de algunas especies de melolonthidos son: 1)Expansión de la frontera agropecuaria y asentamientos humanos. 2) Extensión de redes de comunicación. 3) Alteración de la cobertura vegetal. Estos factores modifican sustancialmente la extensión y estructura de los ecosistemas característicos del país, lo que ha favorecido la dispersión e incremento poblacional de algunas especies, sobre todo aquellas cuyas larvas se puedan adaptar a vastas extensiones de monocultivo de gramíneas. En este contexto, las especies que aparentemente están aprovechando las condiciones de monocultivo de maíz en Uricho y en San Felipe son las del género Phyllophaga. Mientras que las larvas de Macrodactylus sp., Diplotaxis sp., y Anomala sp 1, parecen estar mas asociadas a condiciones propias de vegetación ruderal, especialmente en San Felipe, donde el suelo de las áreas agrícolas permanecen sin movimiento alguno durante 10 meses, además se mantienen con la cobertura de residuos de la cosecha de maíz, vegetación ruderal y aportación de estiércol durante la temporada de pastoreo. En el caso de Uricho, a pesar de que se utiliza la tierra con cultivos de invierno, las condiciones del suelo son, baja perturbación de la capa arable y protección de la superficie por una capa vegetal con especies forrajeras también consideradas como de cobertura, lo que parece estar favoreciendo demasiado a Macrodactylus sp. que aunque a nivel regional su larva no se ha registrado como plaga de maíz o de los cultivos de invierno, en estado adulto está representando un riesgo durante el periodo de floración de las áreas frutícolas y del cultivo de maíz en el Municipio de Erongaricuaro, incluso algunos productores han mencionado la voracidad de estos insectos en su fase adulta durante el periodo de fructificación de manzana. 


\section{AGRADECIMIENTOS}

Agradecemos el apoyo del Instituto de Ecología, A. C. sede Pátzcuaro, Mich., a cargo del Dr. Sergio Zamudio.

\section{LITERATURA CITADA}

Aragón-García A., \& M. A. Morón, 2000. Los coleópteros Melolonthidae asociados a la rizósfera de la caña de azúcar en Chietla, Puebla, México. Folia Entomol.Méx., 108: 7994.

Espinosa-Islas A., M. A. Morón, H. Sánchez, N. Bautista, \& J. Romero, 2005. Complejo gallina ciega (Coleoptera: Melolonthidae) asociado con céspedes en Montecillos, Texcoco, Estado de México. Folia Entomol. Méx., 44(2): 97-107.

Gutiérrez-Nava P., E. Pérez-Agis \& V. Soto-Morales, 2003. Plan de Ordenamiento Territorial Comunitario Comunidad Indígena San Felipe de los Herreros, Charapan, Michoacán. Informe Técnico PAIR, A.C.-COINBIO, Pátzcuaro, Mich.

Instituto Nacional de Estadística Geografía e Informática (INEGI), 2004. Anuario Estadístico Michoacán Ocampo. Agricultura. Gobierno del Estado de Michoacán. México

Magurran A.E., 1987. Ecological Diversity and its Measurement. Princeton University Press. Princeton, New Jersey.

Morón M. A., 1996. Melolonthidae (Coleoptera). Pp. 287-307. In: Llorente-Bousquets J., A. García-Aldrete \& E. González-Soriano (eds). Biodiversidad, taxonomía y biogeografía de artrópodos de México: Hacia una sintesis de su conocimiento. UNAM. México.

Morón M. A. 1997. White grubs (Coleoptera: Melolonthidae: Phyllophaga Harris) in México and Central America. A brief review. Trends Entomol. I :117 - 128.

Nájera-Ricón, M. B. 1998. Diversidad y abundancia del complejo "gallina ciega" (Coleoptera: Melolonthidae) en agroecosistemas de maíz de la región templada de Michoacán, México. Pp.99-106. In: Morón, M. A. \& A. Aragón (eds). Avances en el estudio de la diversidad, importancia y manejo de los Coleópteros edaficolas americanos. Publicación especial de la Benemérita Universidad Autónoma de Puebla y la Sociedad Mexicana de Entomología, A. C. Puebla, México.

Nájera-Rincón M.B. 2005. Control microbiano de "gallina ciega" (Coleoptera: Melolonthidae). Una alternativa para el Manejo Agroecológico de plagas rizófagas. Pp. 101-125. In: Aragón, G.A., J.F. López-Olguín \& A.M. Tapia R. (eds). Manejo Agroecológico de Sistemas. Publicación especial de la Benemérita Universidad Autónoma de Puebla. Puebla, México.

Ramírez-Salinas C., M. A. Morón \& A. Castro 2000. Descripción de los estados inmaduros de seis especies de Phyllophaga (Coleoptera: Melolonthidae) de la región Altos de Chiapas, México. Folia Entomol. Méx., 109: 73-106.

Secretaría de Educación de Michoacán (SEM) 2004. Atlas Geográfico del estado de Michoacán. Universidad Michoacana de San Nicolás de Hidalgo. EDDISA, México.

Recibido: 7 de mayo de 2007

Aceptado: 20 de septiembre de 2007 